CRYSTALLOGRAPHIC COMMUNICATIONS

ISSN 2056-9890

\section{Synthesis, crystal structure, spectroscopic features and Hirshfeld surfaces of 2-methyl-3-[(2-methyl- phenyl)carbamoyl]phenyl acetate}

\author{
Mavișe Yaman, ${ }^{a}$ Șukriye Cakmak, ${ }^{\text {b }}$ Necmi Dege, ${ }^{\text {a }}$ Mustafa Odabașoğlu, ${ }^{c}$ Vadim A. \\ Pavlenko $^{\mathrm{d} *}$ and Halil Kutuk
}

Received 26 October 2018

Accepted 2 January 2019

Edited by A. V. Yatsenko, Moscow State University, Russia

Keywords: crystal structure; amide; X-ray diffraction; Hirshfeld surface; hydrogen bonding.

CCDC reference: 1584572

Supporting information: this article has supporting information at journals.iucr.org/e

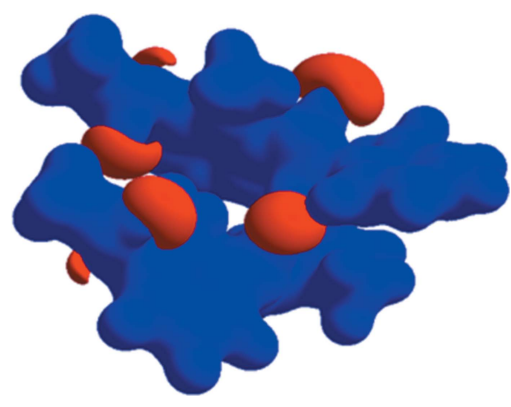

\section{Chemical context}

Amides and their derivatives are extremely important biologically active compounds. Amide groups are present in a number of natural products, polymers and pharmaceuticals (Valeur \& Bradley, 2009; Xiang et al., 2012). Amide derivatives have been found to exhibit biological and pharmacological activities such as antitumor, antimicrobial, antibacterial, antifungal, anti-HSV, analgesic, anti-inflammatory and anticancer (Carbonnelle et al., 2005). Moreover, amide-based compounds represent an important group of efficient chelating ligands (Strotmeyer et al., 2003; Sliva et al., 1997; Pavlishchuk et al., 2011; Gumienna-Kontecka et al., 2007). Recently, we synthesized and studied some new substituted secondary benzamide derivatives obtained as a result of the interaction of aniline-based compounds with acyl chlorides (Çakmak et al., 2016; Kırca et al., 2018; Demir et al., 2015; Kansız, Çakmak et al., 2018). Among them, 3-acetoxy-2methyl- $N$-(4-methoxyphenyl) benzamide was found to exhibit good antioxidant activity (Demir et al., 2015). As a continuation of this work, we prepared the title compound and studied its spectroscopic and structural features.

\section{Structural commentary}

The asymmetric unit of the title compound (Fig. 1) contains two molecules, $A$ and $B$, which adopt different conformations that can be characterized by the mutual arrangement of the acetoxy and terminal 2-methylphenyl groups with respect to 
the plane of the central benzene ring: in molecule $A$ they lie on different sides of this plane, whereas in molecule $B$ they are positioned on the same side. The torsion angles characterizing the conformation details are summarized in Table 1. The dihedral angles subtended by the aromatic rings are 54.33 (12) and $66.68(11)^{\circ}$ in molecules $A$ and $B$, respectively. The molecular conformations are stabilized by weak intramolecular $\mathrm{C}-\mathrm{H} \cdots \mathrm{O}$ contacts (Table 2). All bond lengths and angles are typical of similar compounds, bearing in mind the effect of intermolecular hydrogen bonds on the geometry of the amido groups.<smiles>CC(=O)Oc1cccc(C(=O)Nc2ccccc2C)c1C</smiles>

\section{Supramolecular features}

The packing diagram of the title compounds is presented in Fig. 2. In the crystal, the molecules are linked through strong $\mathrm{N}-\mathrm{H} \cdots \mathrm{O}$ hydrogen bonds (Table 2) into chains along [010]. They are further linked by $\mathrm{C}-\mathrm{H} \cdots \mathrm{O}$ and $\mathrm{C}-\mathrm{H} \cdots \pi$ contacts (Table 2).

\section{Database survey}

A search in the Cambridge Structural Database (CSD version 5.39, update of August 2018; Groom et al., 2016) for 3-acetoxy$N$-phenylbenzamide derivatives gave three hits: 3-acetoxy-2methyl- $N$-(4-methylphenyl)benzamide (HEJBIK; Kirca et al., 2018), 3-acetoxy-2-methyl- $N$-phenylbenzamide and 3-acetoxy2-methyl- $N$-(4-methoxyphenyl)benzamide (HEJBOQ and

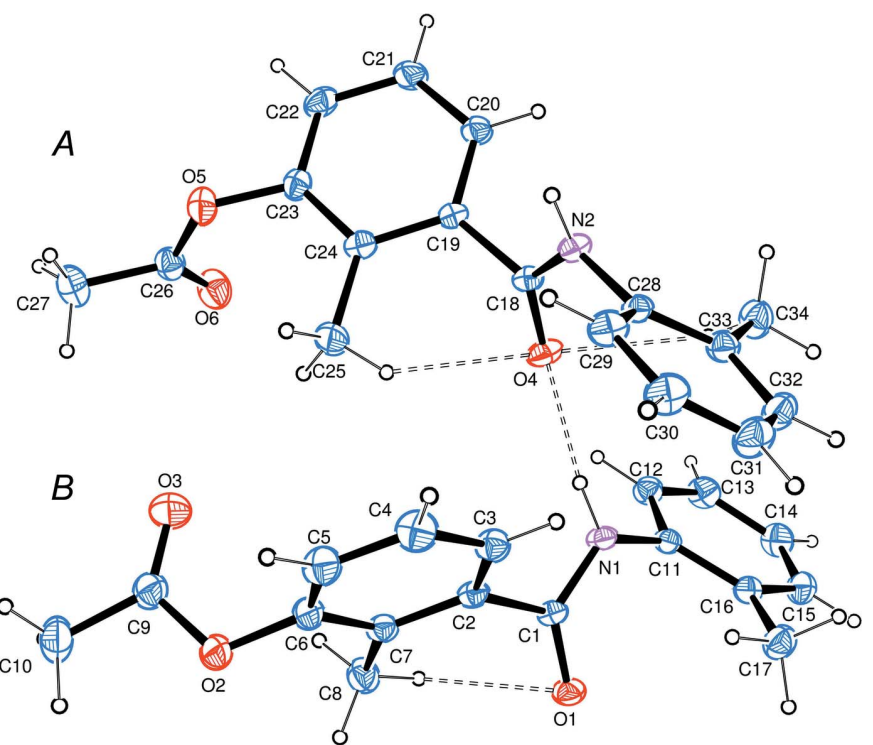

Figure 1

The asymmetric unit of the title compound, with displacement ellipsoids drawn at the $50 \%$ probability level.
Table 1

Selected geometric parameters $\left(\AA,{ }^{\circ}\right)$.

\begin{tabular}{lrlr}
\hline $\mathrm{O} 1-\mathrm{C} 1$ & $1.222(3)$ & $\mathrm{O} 3-\mathrm{C} 9$ & $1.186(4)$ \\
$\mathrm{O} 4-\mathrm{C} 18$ & $1.224(3)$ & $\mathrm{O} 6-\mathrm{C} 26$ & $1.188(4)$ \\
$\mathrm{N} 1-\mathrm{C} 1$ & $1.348(3)$ & $\mathrm{N} 2-\mathrm{C} 18$ & $1.344(4)$ \\
& & & \\
$\mathrm{O} 1-\mathrm{C} 1-\mathrm{N} 1$ & $123.5(3)$ & $\mathrm{C} 1-\mathrm{N} 1-\mathrm{C} 11$ & $123.4(2)$ \\
$\mathrm{O} 4-\mathrm{C} 18-\mathrm{N} 2$ & $123.6(3)$ & $\mathrm{C} 18-\mathrm{N} 2-\mathrm{C} 28$ & $124.2(2)$ \\
& & & \\
$\mathrm{C} 9-\mathrm{O} 2-\mathrm{C} 6-\mathrm{C} 7$ & $-100.0(3)$ & $\mathrm{C} 26-\mathrm{O} 5-\mathrm{C} 23-\mathrm{C} 24$ & $-83.7(3)$ \\
$\mathrm{N} 1-\mathrm{C} 1-\mathrm{C} 2-\mathrm{C} 7$ & $129.1(3)$ & $\mathrm{C} 24-\mathrm{C} 19-\mathrm{C} 18-\mathrm{N} 2$ & $-113.6(3)$ \\
$\mathrm{C} 2-\mathrm{C} 1-\mathrm{N} 1-\mathrm{C} 11$ & $-172.4(2)$ & $\mathrm{C} 28-\mathrm{N} 2-\mathrm{C} 18-\mathrm{C} 19$ & $166.2(2)$ \\
$\mathrm{C} 1-\mathrm{N} 1-\mathrm{C} 11-\mathrm{C} 16$ & $-66.4(4)$ & $\mathrm{C} 18-\mathrm{N} 2-\mathrm{C} 28-\mathrm{C} 33$ & $66.0(4)$ \\
\hline
\end{tabular}

Table 2

Hydrogen-bond geometry $\left(\AA{ }^{\circ}\right)$.

$\mathrm{Cg} 1$ is the centroid of the $\mathrm{C} 28-\mathrm{C} 33$ ring.

\begin{tabular}{lllll}
\hline$D-\mathrm{H} \cdots A$ & $D-\mathrm{H}$ & $\mathrm{H} \cdots A$ & $D \cdots A$ & $D-\mathrm{H} \cdots A$ \\
\hline $\mathrm{C} 5-\mathrm{H} 5 \cdots \mathrm{O} 6^{\mathrm{i}}$ & 0.93 & 2.49 & $3.402(4)$ & 167 \\
$\mathrm{~N} 2-\mathrm{H} 2 \cdots 1^{\mathrm{ii}}$ & $0.88(3)$ & $1.96(3)$ & $2.813(3)$ & $164(2)$ \\
$\mathrm{N} 1-\mathrm{H} 1 \cdots \mathrm{O} 4$ & $0.91(3)$ & $1.91(3)$ & $2.804(3)$ & $166(2)$ \\
$\mathrm{C} 25-\mathrm{H} 25 B \cdots \mathrm{O} 4$ & 0.96 & 2.76 & $3.117(4)$ & 103 \\
$\mathrm{C} 34-\mathrm{H} 34 A \cdots \mathrm{O} 4$ & 0.96 & 2.59 & $3.100(4)$ & 114 \\
$\mathrm{C} 8-\mathrm{H} 8 B \cdots \mathrm{O} 1$ & 0.96 & 2.75 & $2.986(4)$ & 95 \\
$\mathrm{C} 3-\mathrm{H} 3 \cdots \mathrm{Cg} 1$ & 0.93 & 2.81 & $3.666(3)$ & 153 \\
\hline
\end{tabular}

Symmetry codes: (i) $x-1, y, z$; (ii) $x, y+1, z$.

JUMCEB, respectively; both Demir et al., 2015). The structure of HEJBIK is especially close to that of the title compound: it also contains two molecules in an asymmetric unit and is isostructural to the title compound with the exception of one methyl group (2-Me in the title compound and 4-Me in HEJBIK). The two independent molecules in HEJBIK have different conformations in the same manner, as in the title structure. In the two structures HEJBOQ and JUMCEB, the acetoxy groups and the terminal benzene rings are positioned on opposite sides of the planes formed by the central benzene rings. In all these structures, the molecules are linked into chains by $\mathrm{N}-\mathrm{H} \cdots \mathrm{O}$ hydrogen bonds.

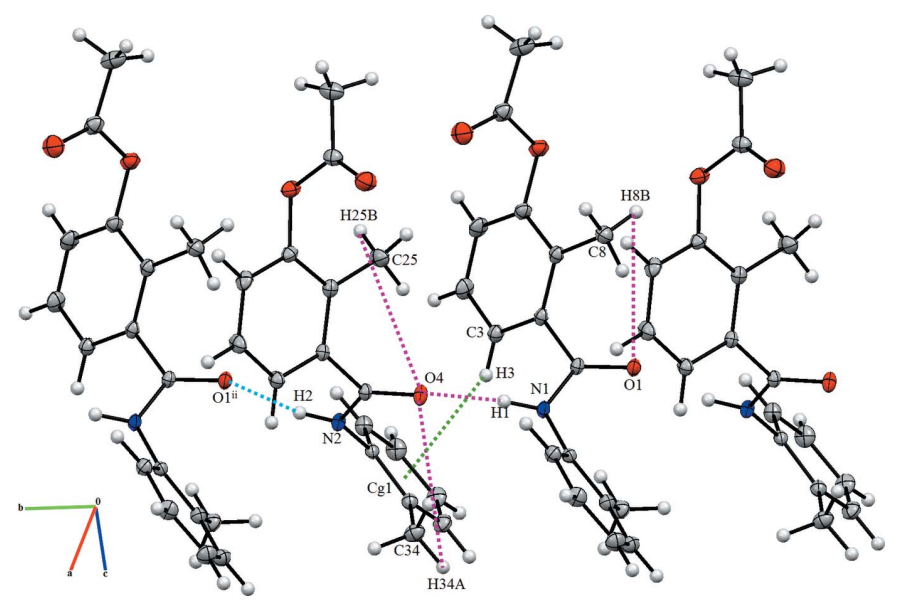

Figure 2

Packing diagram of the title compound showing the short intermolecular contacts. $\mathrm{Cg} 1$ is the centroid of the $\mathrm{C} 28-\mathrm{C} 33$ benzene ring. 


\section{Hirshfeld surface analysis}

The molecular Hirshfeld surfaces $\left(d_{\text {norm }}\right)$ for molecules $A$ and $B$ of the title compound generated using CrystalExplorer3.1 (Wolff et al., 2012) and are presented in Fig. 3. The $d_{\text {norm }}$ values are mapped on the Hirshfeld surfaces using a red-blue-white colour scheme (Spackman \& Jayatilaka, 2009) as follows: the dark-red spots indicate the closest contacts related to the $\mathrm{N}-$ $\mathrm{H}$... O hydrogen bonds, the other short intermolecular contacts appear as light-red spots, blue regions depict positive $d_{\text {norm }}$ values, and in the white regions the lengths of the contacts are exactly equal to the sum of van der Waals radii $\left(d_{\text {norm }}=0\right)$. Analogous dark-red spots related to the $\mathrm{N}-$ $\mathrm{H}$... O interactions were observed on the Hirshfeld surfaces of similar molecules (Şen et al., 2017; Gümüş et al., 2018; Kansız $\&$ Dege, 2018). Figs. 4 and 5 show the two-dimensional fingerprint plots for molecules $A$ and $B$, respectively. For both molecules, the contributions from the $\mathrm{H} \cdots \mathrm{H} / \mathrm{H} \cdots \mathrm{H}$ contacts are the largest (55.3 and $53.9 \%$ for $A$ and $\mathrm{B}$, respectively). The

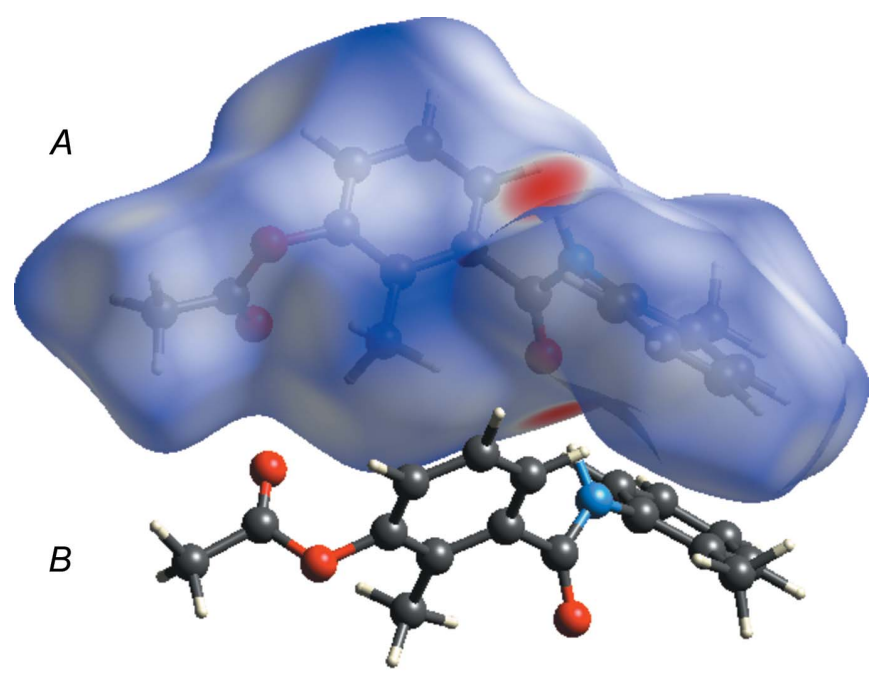

(a)

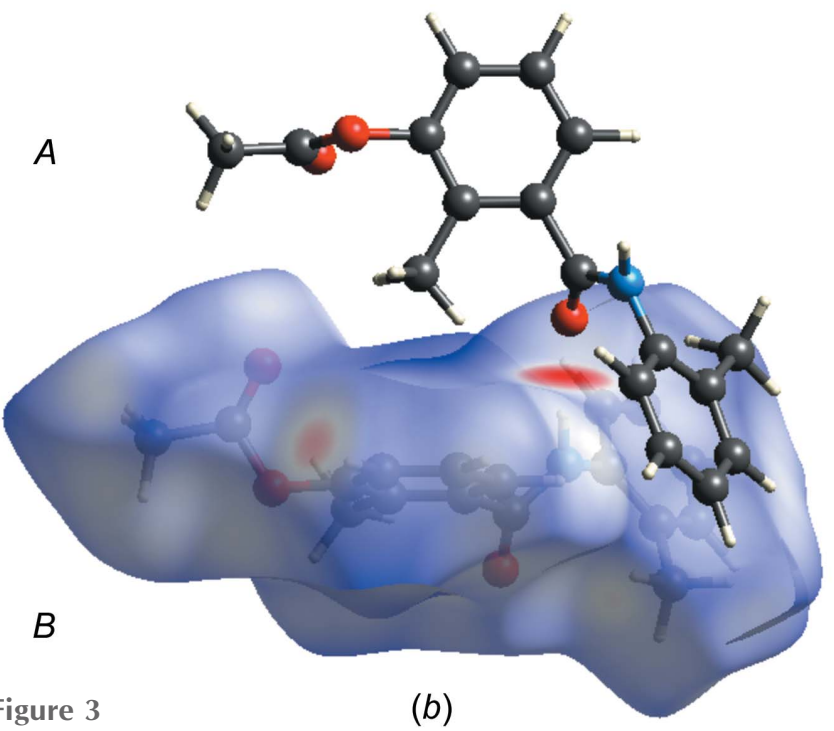

Figure 3

(b)

Hirsfeld surfaces of 3-acetoxy-2-methyl- $N$-(3-methylphenyl) benzamide (three-dimensional $d_{\text {norm }}$ surface): ( $a$ ) molecule $A$ and (b) molecule $B$.
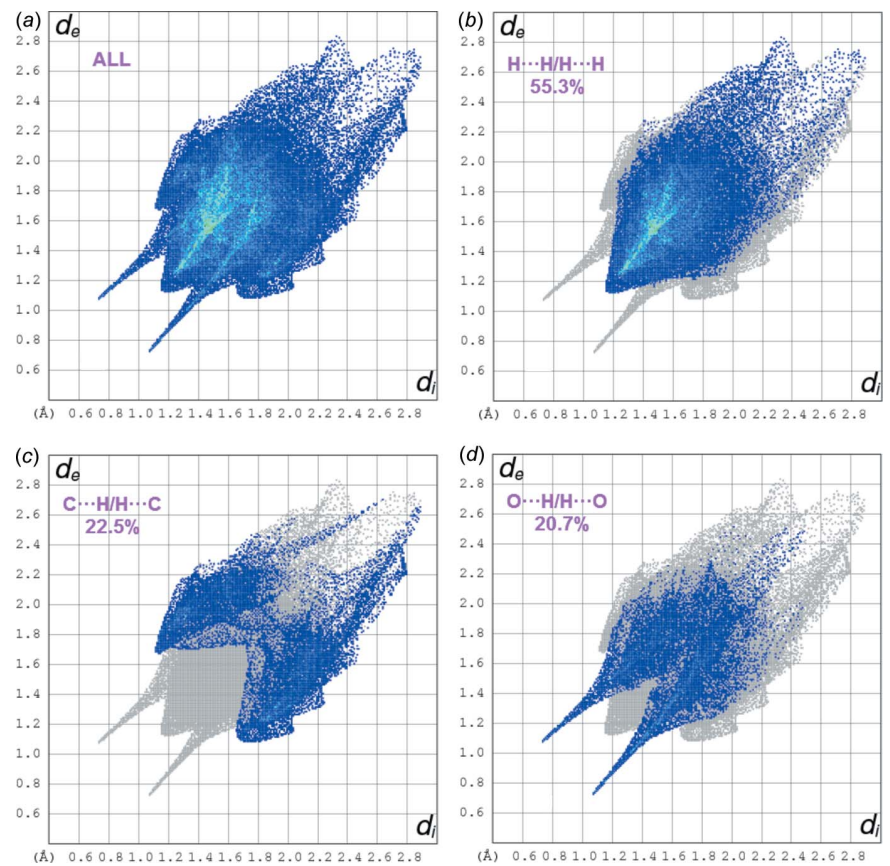

Figure 4

The fingerprint plots for molecule $A$ : $(a)$ all atoms inside $\cdot$ all atoms outside (100\%), (b) $\mathrm{H}_{\text {inside }} \cdots \mathrm{H}_{\text {outside }} / \mathrm{H}_{\text {outside }} \cdots \mathrm{H}_{\text {inside }}(55.3 \%)$, (c) $\mathrm{C}_{\text {inside }} \cdots$ $\mathrm{H}_{\text {outside }} / \mathrm{H}_{\text {outside }} \cdots \mathrm{C}_{\text {inside }}(22.5 \%)$ and $(d) \quad \mathrm{O}_{\text {inside }} \cdots \mathrm{H}_{\text {outside }} / \mathrm{H}_{\text {outside }} \cdots$ $\mathrm{O}_{\text {inside }}(20.7 \%)$.

contributions of the other intermolecular contacts are as follows: $\mathrm{C} \cdots \mathrm{H} / \mathrm{H} \cdots \mathrm{C}(22.5 \%)$ and $\mathrm{O} \cdots \mathrm{H} / \mathrm{H} \cdots \mathrm{O}(20.7 \%)$ for $A$ and $\mathrm{C} \cdots \mathrm{H} / \mathrm{H} \cdots \mathrm{C}(23.8 \%)$ and $\mathrm{O} \cdots \mathrm{H} / \mathrm{H} \cdots \mathrm{O}(21.7 \%)$ for $B$. The Hirshfeld surface mapped over the electrostatic potential
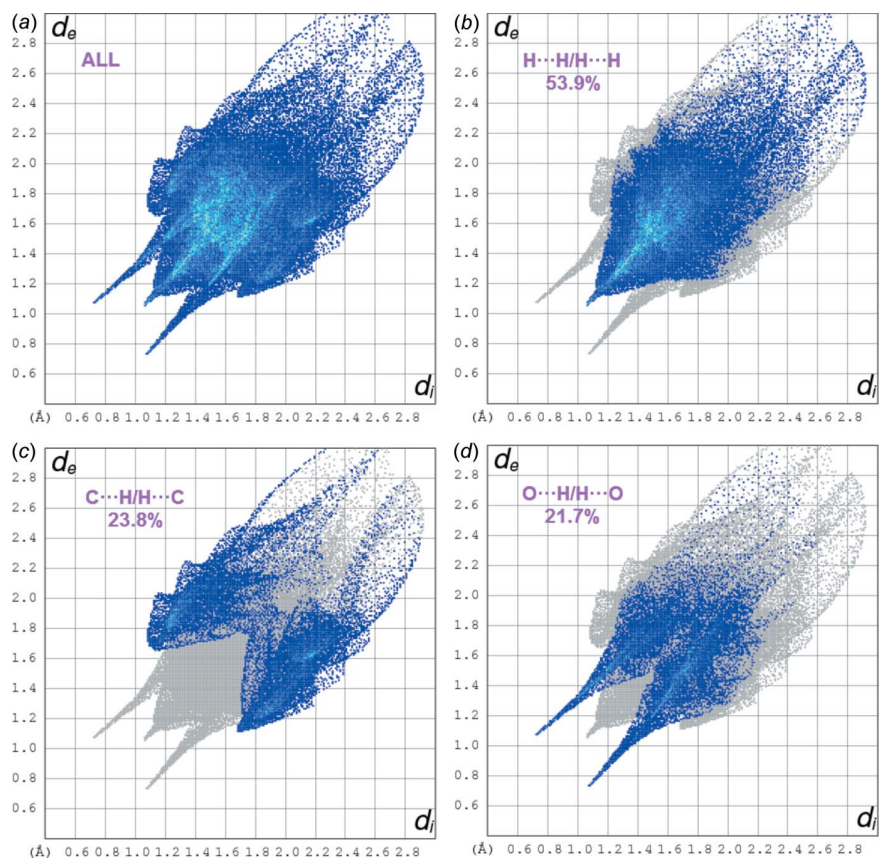

Figure 5

The fingerprint plots for molecule $B:(a)$ all atoms inside $\cdots$ all atoms $\mathrm{sutside}_{\text {oute }}$ (100\%), (b) $\mathrm{H}_{\text {inside }} \cdots \mathrm{H}_{\text {outside }} / \mathrm{H}_{\text {outside }} \cdots \mathrm{H}_{\text {inside }}$ (53.9\%), (c) $\mathrm{C}_{\text {inside }} \cdots$ $\mathrm{H}_{\text {outside }} / \mathrm{H}_{\text {outside }} \cdots \mathrm{C}_{\text {inside }}(23.8 \%)$ and (d) $\mathrm{O}_{\text {inside }} \cdots \mathrm{H}_{\text {outside }} / \mathrm{H}_{\text {outside }} \cdots$ $\mathrm{O}_{\text {inside }}(21.7 \%)$. 


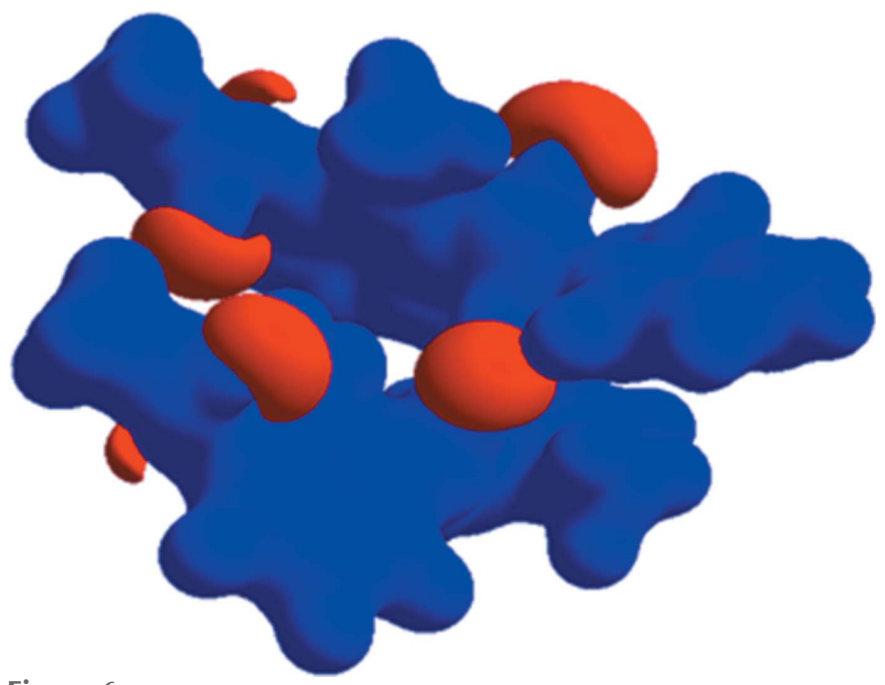

Figure 6

Electrostatic potential mapped on the Hirshfeld surface ( \pm 0.25 a.u.).

$\mathrm{n}( \pm 0.25$ a.u. $)$ is shown in Fig. 6 where blue regions correspond to positive electrostatic potential and red spots related to the oxygen atoms represent the areas of negative electrostatic potential; the distribution is analogous to that in a similar compound (Yaman et al., 2018).

\section{Vibrational spectrum}

The IR spectrum of the title compound $\left(\mathrm{KBr}, \mathrm{cm}^{-1}\right)$ shown in Fig. 7 exhibits the following characteristic bands: $3210(\mathrm{~N}-\mathrm{H})$, 1761 (acetoxy $\mathrm{C}=\mathrm{O}$ ), 1651 (amide $\mathrm{C}=\mathrm{O}$ ). Because of the interaction of the aromatic group with the acetoxy carbonyl moiety, the frequency of the acetoxy $\mathrm{C}=\mathrm{O}$ stretching vibration is larger compared to the normal frequency of the stretching vibrations in esters $\left(1740 \mathrm{~cm}^{-1}\right)$.

\section{Synthesis and crystallization}

The synthesis was performed according to the reaction scheme presented in Fig. 8 and applied earlier for the synthesis of analogous compounds (Cakmak et al., 2016; Kirca et al., 2018, Demir et al., 2015). A solution of 3-acetoxy-2-methylbenzoyl

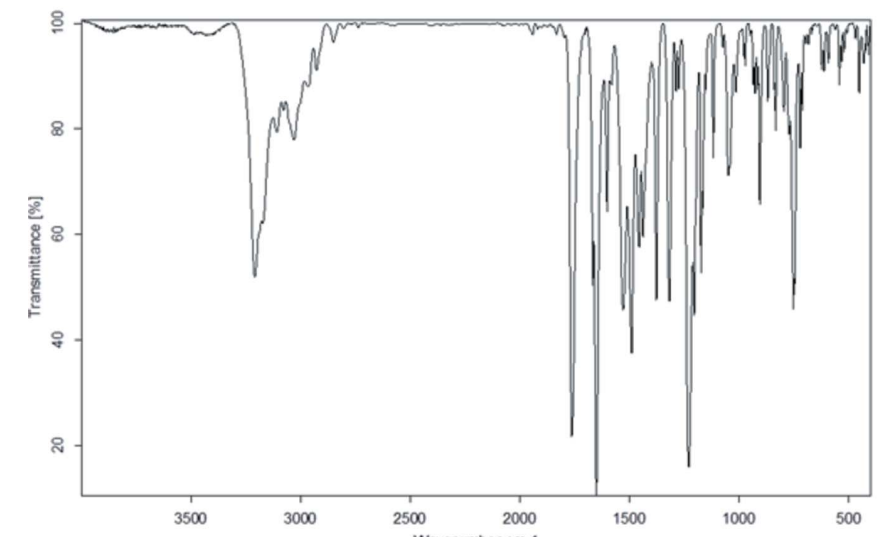

Figure 7

IR spectrum of the title compound.
Table 3

Experimental details.

Crystal data

Chemical formula

$M_{\mathrm{r}}$

Crystal system, space group

Temperature (K)

$a, b, c(\AA)$

$\alpha, \beta, \gamma\left({ }^{\circ}\right)$

$V\left(\AA^{3}\right)$

$Z$

Radiation type

$\mu\left(\mathrm{mm}^{-1}\right)$

Crystal size (mm)

$\mathrm{C}_{17} \mathrm{H}_{17} \mathrm{NO}_{3}$

283.31

Triclinic, $P \overline{1}$

296

7.7842 (5), 8.8802 (5), 22.2112 (15)

94.791 (5), 97.620 (5), 90.043 (5)

1516.37 (17)

Mo $K \alpha$

0.09

Data collection

Diffractometer

Absorption correction

$T_{\min }, T_{\max }$

No. of measured, independent and observed $[I>2 \sigma(I)]$ reflections

$R_{\text {in }}$

$(\sin \theta / \lambda)_{\max }\left(\AA^{-1}\right)$

$0.42 \times 0.37 \times 0.21$

Stoe IPDS 2

Integration ( $X$-RED32; Stoe \& Cie, 2002)

$0.958,0.993$

21781, 5950, 3029

0.086

0.617

Refinement

$R\left[F^{2}>2 \sigma\left(F^{2}\right)\right], w R\left(F^{2}\right), S$

No. of reflections

No. of parameters

$\mathrm{H}$-atom treatment

$0.057,0.159,0.90$

5950

393

$\mathrm{H}$ atoms treated by a mixture of independent and constrained refinement

$\Delta \rho_{\max }, \Delta \rho_{\min }\left(\mathrm{e} \AA^{-3}\right)$ $0.17,-0.14$

Computer programs: $X$-AREA and X-RED32 (Stoe \& Cie, 2002), SHELXT (Sheldrick, 2015a), SHELXL2017 (Sheldrick, 2015b), ORTEP-3 for Windows and Win GX (Farrugia, 2012), PLATON (Spek, 2009) and publCIF (Westrip, 2010).

chloride $(11 \mathrm{mmol})$ in THF $(10 \mathrm{~mL})$ was added dropwise to a solution of 2-methylaniline $(10 \mathrm{mmol})$ and triethylamine $(10 \mathrm{mmol})$ in THF $(10 \mathrm{~mL})$ at room temperature. After the reaction mixture had been stirred at room temperature for $15 \mathrm{~h}$, the resulting white precipitate was filtered off and then $100 \mathrm{ml}$ of water was added dropwise to the filtrate. The precipitate was filtered off and washed several times with water to remove the unreacted reagents and triethylamine hydrochloride. The crude product was recrystallized from acetonitrile (1.82 g, 58\%; m.p. 435-438 K). Single crystals were obtained from an acetonitrile solution after incubation in the fridge for 20 days.

\section{Refinement}

Crystal data, data collection and structure refinement details are summarized in Table 3. The $\mathrm{N}$-bound $\mathrm{H}$ atoms were freely refined. C-bound hydrogen atoms were positioned geom-

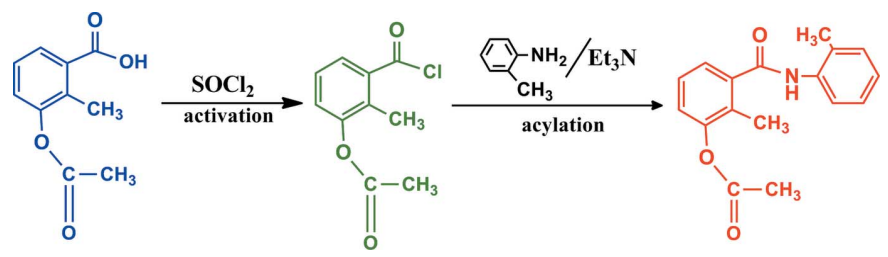

Figure 8

Reaction scheme. 
etrically and refined as riding with $\mathrm{C}-\mathrm{H}=0.93 \AA$ and $U_{\text {iso }}(\mathrm{H})$ $=1.2 U_{\text {eq }}(\mathrm{C})$ for aromatic $\mathrm{C}$ atoms and $\mathrm{C}-\mathrm{H}=0.96 \AA$ and $U_{\text {iso }}(\mathrm{H})=1.5 U_{\text {eq }}(\mathrm{C})$ for methyl groups. Each methyl group was allowed to rotate about its parent $\mathrm{C}-\mathrm{C}$ bond.

\section{Acknowledgements}

The authors acknowledge the Faculty of Arts and Sciences, Ondokuz Mayis University, Turkey, for the use of the Stoe IPDS 2 diffractometer (purchased under grant F.279 of the University Research Fund).

\section{References}

Cakmak, S., Kutuk, H., Odabasoglu, M., Yakan, H. \& Buyukgungor, O. (2016). Lett. Org. Chem. 13, 181-194.

Carbonnelle, D., Ebstein, F., Rabu, C., Petit, J. Y., Gregoire, M. \& Lang, F. (2005). Eur. J. Immunol. 35, 546-556.

Demir, S., Cakmak, S., Dege, N., Kutuk, H., Odabasoglu, M. \& Kepekci, R. A. (2015). J. Mol. Struct. 1100, 582-591.

Farrugia, L. J. (2012). J. Appl. Cryst. 45, 849-854.

Groom, C. R., Bruno, I. J., Lightfoot, M. P. \& Ward, S. C. (2016). Acta Cryst. B72, 171-179.

Gumienna-Kontecka, E., Golenya, I. A., Dudarenko, N. M., Dobosz, A., Haukka, M., Fritsky, I. O. \& Świątek-Kozłowska, J. (2007). New J. Chem. 31, 1798-1805.

Gümüs,, M. K., Kansız, S., Aydemir, E., Gorobets, N. Y. \& Dege, N. (2018). J. Mol. Struct. 1168, 280-290.
Kansız, S., Çakmak, Ş., Dege, N., Meral, G. \& Kütük, H. (2018). XRay Struct. Anal. Online, 34, 17-18.

Kansiz, S. \& Dege, N. (2018). J. Mol. Struct. 1173, 42-51.

Kırca, B. K., Çakmak, Ş., Kütük, H., Odabaşoğlu, M. \& Büyükgüngör, O. (2018). J. Mol. Struct. 1151, 191-197.

Pavlishchuk, A. V., Kolotilov, S. V., Zeller, M., Shvets, O. V., Fritsky, I. O., Lofland, S. E., Addison, A. W. \& Hunter, A. D. (2011). Eur. J. Inorg. Chem. pp. 4826-4836.

Şen, F., Kansiz, S. \& Uçar, İ. (2017). Acta Cryst. C73, 517-524.

Sheldrick, G. M. (2015a). Acta Cryst. A71, 3-8.

Sheldrick, G. M. (2015b). Acta Cryst. C71, 3-8.

Sliva, T. Yu., Duda, A. M., Głowiak, T., Fritsky, I. O., Amirkhanov, V. M., Mokhir, A. A. \& Kozłowski, H. (1997). J. Chem. Soc. Dalton Trans. pp. 273-276.

Spackman, M. A. \& Jayatilaka, D. (2009). CrystEngComm, 11, 19-32. Spek, A. L. (2009). Acta Cryst. D65, 148-155.

Stoe \& Cie (2002). $X$-AREA and X-RED32. Stoe \& Cie GmbH, Darmstadt, Germany.

Strotmeyer, K. P., Fritsky, I. O., Ott, R., Pritzkow, H. \& Krämer, R. (2003). Supramol. Chem. 15, 529-547.

Valeur, E. \& Bradley, M. (2009). Chem. Soc. Rev. 38, 606-631.

Westrip, S. P. (2010). J. Appl. Cryst. 43, 920-925.

Wolff, S. K., Grimwood, D. J., McKinnon, J. J., Turner, M. J., Jayatilaka, D. \& Spackman, M. A. (2012). CrystalExplorer3.1. University of Western Australia.

Xiang, Y.-F., Qian, C.-W., Xing, G.-W., Hao, J., Xia, M. \& Wang, Y.-F. (2012). Bioorg. Med. Chem. Lett. 22, 4703-4706.

Yaman, M., Almarhoon, Z. M., Çakmak, Ş., Kütük, H., Meral, G. \& Dege, N. (2018). Acta Cryst. E74, 41-44. 


\section{supporting information}

Acta Cryst. (2019). E75, 423-427 [https://doi.org/10.1107/S2056989019000021]

Synthesis, crystal structure, spectroscopic features and Hirshfeld surfaces of 2methyl-3-[(2-methylphenyl)carbamoyl]phenyl acetate

Mavişe Yaman, Şukriye Cakmak, Necmi Dege, Mustafa Odabaşoğlu, Vadim A. Pavlenko and Halil Kutuk

Computing details

Data collection: $X$-AREA (Stoe \& Cie, 2002); cell refinement: $X$-AREA (Stoe \& Cie, 2002); data reduction: $X$-RED32

(Stoe \& Cie, 2002); program(s) used to solve structure: SHELXT (Sheldrick, 2015a); program(s) used to refine structure: SHELXL2017 (Sheldrick, 2015b); molecular graphics: ORTEP-3 for Windows (Farrugia, 2012); software used to prepare material for publication: WinGX (Farrugia, 2012), PLATON (Spek, 2009) and publCIF (Westrip, 2010).

2-Methyl-3-[(2-methylphenyl)carbamoyl] phenyl acetate

Crystal data

$\mathrm{C}_{17} \mathrm{H}_{17} \mathrm{NO}_{3}$

$M_{r}=283.31$

Triclinic, $P \overline{1}$

$a=7.7842(5) \AA$

$b=8.8802(5) \AA$

$c=22.2112(15) \AA$

$\alpha=94.791(5)^{\circ}$

$\beta=97.620(5)^{\circ}$

$\gamma=90.043(5)^{\circ}$

$V=1516.37(17) \AA^{3}$

\section{Data collection}

Stoe IPDS 2 diffractometer

Radiation source: sealed X-ray tube, $12 \times 0.4$ $\mathrm{mm}$ long-fine focus

Detector resolution: 6.67 pixels $\mathrm{mm}^{-1}$

rotation method scans

Absorption correction: integration

(X-RED32; Stoe \& Cie, 2002)

$T_{\text {min }}=0.958, T_{\text {max }}=0.993$

\section{Refinement}

Refinement on $F^{2}$

Least-squares matrix: full

$R\left[F^{2}>2 \sigma\left(F^{2}\right)\right]=0.057$

$w R\left(F^{2}\right)=0.159$

$S=0.90$

5950 reflections
$Z=4$

$F(000)=600$

$D_{\mathrm{x}}=1.241 \mathrm{Mg} \mathrm{m}^{-3}$

Mo $K \alpha$ radiation, $\lambda=0.71073 \AA$

Cell parameters from 19688 reflections

$\theta=1.9-27.5^{\circ}$

$\mu=0.09 \mathrm{~mm}^{-1}$

$T=296 \mathrm{~K}$

Prism, colorless

$0.42 \times 0.37 \times 0.21 \mathrm{~mm}$

21781 measured reflections

5950 independent reflections

3029 reflections with $I>2 \sigma(I)$

$R_{\text {int }}=0.086$

$\theta_{\max }=26.0^{\circ}, \theta_{\min }=1.9^{\circ}$

$h=-9 \rightarrow 9$

$k=-10 \rightarrow 10$

$l=-27 \rightarrow 27$

\section{3 parameters}

0 restraints

Hydrogen site location: mixed

$\mathrm{H}$ atoms treated by a mixture of independent and constrained refinement 
$w=1 /\left[\sigma^{2}\left(F_{\mathrm{o}}^{2}\right)+(0.0763 P)^{2}\right]$

where $P=\left(F_{\mathrm{o}}^{2}+2 F_{\mathrm{c}}^{2}\right) / 3$

$(\Delta / \sigma)_{\max }<0.001$

$$
\begin{aligned}
\Delta \rho_{\max } & =0.17 \text { e } \AA^{-3} \\
\Delta \rho_{\text {m. }} & =-0.13 \text { e } \AA^{-3}
\end{aligned}
$$

\section{Special details}

Geometry. All esds (except the esd in the dihedral angle between two 1.s. planes) are estimated using the full covariance matrix. The cell esds are taken into account individually in the estimation of esds in distances, angles and torsion angles; correlations between esds in cell parameters are only used when they are defined by crystal symmetry. An approximate (isotropic) treatment of cell esds is used for estimating esds involving l.s. planes.

Fractional atomic coordinates and isotropic or equivalent isotropic displacement parameters $\left(\hat{A}^{2}\right)$

\begin{tabular}{|c|c|c|c|c|}
\hline & $x$ & $y$ & $z$ & $U_{\text {iso }} * / U_{\text {eq }}$ \\
\hline $\mathrm{O} 1$ & $0.5356(3)$ & $0.3278(2)$ & $0.28533(9)$ & $0.0605(6)$ \\
\hline $\mathrm{N} 2$ & $0.6781(3)$ & 1.0533 & $0.32141(11)$ & $0.0482(6)$ \\
\hline $\mathrm{O} 2$ & $0.2232(3)$ & 0.5083 & $0.07897(10)$ & $0.0717(6)$ \\
\hline $\mathrm{C} 1$ & $0.5516(3)$ & 0.4634 & $0.28130(12)$ & $0.0448(7)$ \\
\hline N1 & $0.6771(3)$ & 0.5500 & $0.31509(11)$ & $0.0486(6)$ \\
\hline O5 & $0.7722(3)$ & $1.0738(3)$ & $0.07181(9)$ & $0.0662(6)$ \\
\hline $\mathrm{C} 28$ & $0.5682(4)$ & $0.9934(3)$ & $0.36083(12)$ & $0.0468(7)$ \\
\hline $\mathrm{O} 4$ & $0.7921(3)$ & $0.8326(2)$ & $0.28774(10)$ & $0.0608(6)$ \\
\hline C19 & $0.8414(3)$ & $1.0503(3)$ & $0.23666(12)$ & $0.0438(6)$ \\
\hline $\mathrm{C} 18$ & $0.7690(3)$ & $0.9681(3)$ & $0.28425(13)$ & $0.0464(7)$ \\
\hline $\mathrm{C} 11$ & $0.8180(3)$ & 0.4903 & $0.35334(12)$ & $0.0463(7)$ \\
\hline $\mathrm{C} 16$ & $0.7951(4)$ & $0.4184(3)$ & $0.40468(13)$ & $0.0537(7)$ \\
\hline $\mathrm{C} 2$ & $0.4280(3)$ & $0.5450(3)$ & $0.23801(13)$ & $0.0452(7)$ \\
\hline $\mathrm{C} 3$ & $0.3430(4)$ & $0.6702(3)$ & $0.26034(15)$ & $0.0567(8)$ \\
\hline $\mathrm{H} 3$ & 0.368858 & 0.706558 & 0.301043 & $0.068^{*}$ \\
\hline $\mathrm{C} 12$ & $0.9848(4)$ & $0.5130(3)$ & $0.33845(14)$ & $0.0572(8)$ \\
\hline H12 & 0.999896 & 0.565609 & 0.304998 & $0.069^{*}$ \\
\hline $\mathrm{C} 7$ & $0.3944(4)$ & 0.4892 & $0.17655(14)$ & $0.0516(7)$ \\
\hline $\mathrm{C} 17$ & $0.6204(4)$ & $0.4057(4)$ & $0.42552(15)$ & $0.0705(9)$ \\
\hline H17A & 0.558650 & 0.319989 & 0.403650 & $0.106^{*}$ \\
\hline H17B & 0.555999 & 0.495828 & 0.417841 & $0.106^{*}$ \\
\hline $\mathrm{H} 17 \mathrm{C}$ & 0.634656 & 0.393102 & 0.468398 & $0.106^{*}$ \\
\hline $\mathrm{C} 20$ & $0.9676(4)$ & $1.1600(3)$ & $0.25396(14)$ & $0.0560(8)$ \\
\hline $\mathrm{H} 20$ & 1.006541 & 1.184171 & 0.295057 & $0.067^{*}$ \\
\hline C6 & $0.2705(4)$ & $0.5654(4)$ & $0.14029(14)$ & $0.0585(8)$ \\
\hline $\mathrm{C} 24$ & $0.7774(4)$ & $1.0137(3)$ & $0.17529(14)$ & $0.0532(7)$ \\
\hline C34 & $0.8224(4)$ & $0.8962(4)$ & $0.42816(16)$ & $0.0729(9)$ \\
\hline $\mathrm{H} 34 \mathrm{~A}$ & 0.864042 & 0.813155 & 0.403828 & $0.109^{*}$ \\
\hline H34B & 0.842987 & 0.876968 & 0.470381 & $0.109^{*}$ \\
\hline $\mathrm{H} 34 \mathrm{C}$ & 0.882266 & 0.987300 & 0.422249 & $0.109^{*}$ \\
\hline $\mathrm{C} 23$ & $0.8470(4)$ & $1.0938(3)$ & $0.13335(13)$ & $0.0545(8)$ \\
\hline O6 & $0.9365(4)$ & $0.8729(3)$ & $0.05401(12)$ & $0.0885(8)$ \\
\hline $\mathrm{C} 33$ & $0.6320(4)$ & $0.9139(3)$ & $0.40945(14)$ & $0.0550(7)$ \\
\hline $\mathrm{C} 13$ & $1.1258(4)$ & $0.4580(4)$ & $0.37307(17)$ & $0.0728(10)$ \\
\hline H13 & 1.236428 & 0.472783 & 0.362982 & $0.087 *$ \\
\hline $\mathrm{C} 22$ & $0.9755(4)$ & $1.2010(4)$ & $0.14968(15)$ & $0.0639(9)$ \\
\hline
\end{tabular}




\begin{tabular}{|c|c|c|c|c|}
\hline $\mathrm{H} 22$ & 1.020909 & 1.250648 & 0.119977 & $0.077^{*}$ \\
\hline $\mathrm{C} 26$ & $0.8233(5)$ & $0.9545(4)$ & $0.03635(15)$ & $0.0646(9)$ \\
\hline $\mathrm{C} 4$ & $0.2194(4)$ & $0.7415(4)$ & $0.22218(18)$ & $0.0727(10)$ \\
\hline $\mathrm{H} 4$ & 0.161175 & 0.824827 & 0.237254 & $0.087^{*}$ \\
\hline $\mathrm{C} 32$ & $0.5113(5)$ & $0.8545(4)$ & $0.44225(15)$ & $0.0713(9)$ \\
\hline H32 & 0.550319 & 0.797517 & 0.474527 & $0.086^{*}$ \\
\hline $\mathrm{C} 29$ & $0.3907(4)$ & $1.0207(3)$ & $0.34776(15)$ & $0.0608(8)$ \\
\hline $\mathrm{H} 29$ & 0.350054 & 1.078584 & 0.315939 & $0.073^{*}$ \\
\hline $\mathrm{C} 5$ & 0.1834 (4) & $0.6884(4)$ & 0.16207 (18) & $0.0736(10)$ \\
\hline H5 & 0.100416 & 0.735476 & 0.136156 & $0.088^{*}$ \\
\hline $\mathrm{C} 15$ & $0.9407(4)$ & $0.3628(4)$ & $0.43830(15)$ & $0.0678(9)$ \\
\hline H15 & 0.928027 & 0.311797 & 0.472381 & $0.081^{*}$ \\
\hline $\mathrm{C} 21$ & $1.0363(4)$ & $1.2343(4)$ & $0.21012(16)$ & $0.0680(9)$ \\
\hline $\mathrm{H} 21$ & 1.123516 & 1.306624 & 0.221625 & $0.082^{*}$ \\
\hline C9 & $0.2956(5)$ & $0.5755(5)$ & $0.03514(16)$ & $0.0724(10)$ \\
\hline $\mathrm{C} 31$ & $0.3375(5)$ & $0.8771(4)$ & $0.42858(17)$ & $0.0758(10)$ \\
\hline $\mathrm{H} 31$ & 0.260317 & 0.834551 & 0.451079 & $0.091^{*}$ \\
\hline $\mathrm{C} 14$ & $1.1044(5)$ & $0.3818(4)$ & $0.42210(17)$ & $0.0739(10)$ \\
\hline H14 & 1.200093 & 0.342190 & 0.444874 & $0.089^{*}$ \\
\hline $\mathrm{C} 25$ & $0.6354(5)$ & $0.8978(4)$ & $0.15608(16)$ & $0.0806(11)$ \\
\hline $\mathrm{H} 25 \mathrm{~A}$ & 0.568073 & 0.923610 & 0.119042 & $0.121^{*}$ \\
\hline $\mathrm{H} 25 \mathrm{~B}$ & 0.562053 & 0.895667 & 0.187499 & $0.121^{*}$ \\
\hline $\mathrm{H} 25 \mathrm{C}$ & 0.685593 & 0.800058 & 0.149429 & $0.121^{*}$ \\
\hline $\mathrm{O} 3$ & $0.4029(4)$ & $0.6720(3)$ & $0.04766(13)$ & $0.1018(9)$ \\
\hline C30 & $0.2768(4)$ & $0.9620(4)$ & $0.38199(17)$ & $0.0752(10)$ \\
\hline $\mathrm{H} 30$ & 0.158693 & 0.979873 & 0.373513 & $0.090^{*}$ \\
\hline $\mathrm{C} 8$ & $0.4864(5)$ & $0.3554(4)$ & $0.15108(15)$ & $0.0709(9)$ \\
\hline H8A & 0.432986 & 0.264201 & 0.160420 & $0.106^{*}$ \\
\hline H8B & 0.605821 & 0.358900 & 0.168815 & $0.106^{*}$ \\
\hline $\mathrm{H} 8 \mathrm{C}$ & 0.479238 & 0.357183 & 0.107663 & $0.106^{*}$ \\
\hline $\mathrm{C} 10$ & $0.2217(5)$ & $0.5106(5)$ & $-0.02652(16)$ & $0.0945(13)$ \\
\hline $\mathrm{H} 10 \mathrm{~A}$ & 0.118483 & 0.563994 & -0.040154 & $0.142^{*}$ \\
\hline H10B & 0.193802 & 0.405789 & -0.025063 & $0.142^{*}$ \\
\hline $\mathrm{H} 10 \mathrm{C}$ & 0.304914 & 0.520111 & -0.054284 & $0.142^{*}$ \\
\hline $\mathrm{C} 27$ & $0.7180(5)$ & $0.9428(5)$ & $-0.02461(16)$ & $0.0866(11)$ \\
\hline $\mathrm{H} 27 \mathrm{~A}$ & 0.778041 & 0.882794 & -0.052970 & $0.130^{*}$ \\
\hline $\mathrm{H} 27 \mathrm{~B}$ & 0.699550 & 1.042040 & -0.038207 & $0.130^{*}$ \\
\hline $\mathrm{H} 27 \mathrm{C}$ & 0.608231 & 0.896027 & -0.022119 & $0.130^{*}$ \\
\hline $\mathrm{H} 2$ & $0.653(3)$ & $1.146(3)$ & $0.3128(11)$ & $0.043(7)^{*}$ \\
\hline H1 & $0.696(3)$ & $0.644(3)$ & $0.3039(12)$ & $0.050(8) *$ \\
\hline
\end{tabular}

Atomic displacement parameters $\left(\AA^{2}\right)$

\begin{tabular}{lllllll}
\hline & $U^{11}$ & $U^{22}$ & $U^{33}$ & $U^{12}$ & $U^{13}$ & $U^{23}$ \\
\hline O1 & $0.0662(13)$ & $0.0394(12)$ & $0.0731(14)$ & $-0.0031(10)$ & $-0.0063(10)$ & $0.0143(10)$ \\
N2 & $0.0520(14)$ & $0.0373(13)$ & $0.0590(15)$ & $0.0039(11)$ & $0.0162(12)$ & $0.0120(12)$ \\
O2 & $0.0670(14)$ & $0.0830(16)$ & $0.0625(15)$ & $-0.0198(12)$ & $-0.0088(11)$ & $0.0188(12)$ \\
C1 & $0.0410(15)$ & $0.0385(16)$ & $0.0560(17)$ & $-0.0008(12)$ & $0.0073(13)$ & $0.0098(13)$
\end{tabular}




\begin{tabular}{|c|c|c|c|c|c|c|}
\hline N1 & 0.0473 (14) & $0.0361(13)$ & $0.0616(15)$ & $-0.0058(11)$ & $-0.0006(11)$ & $0.0121(12)$ \\
\hline $\mathrm{O} 5$ & $0.0718(14)$ & $0.0768(15)$ & $0.0500(13)$ & $0.0150(12)$ & $0.0071(11)$ & $0.0076(11)$ \\
\hline $\mathrm{C} 28$ & $0.0541(17)$ & $0.0373(15)$ & $0.0503(17)$ & $-0.0026(13)$ & $0.0124(13)$ & $0.0025(13)$ \\
\hline $\mathrm{O} 4$ & $0.0714(14)$ & $0.0373(11)$ & $0.0799(15)$ & $0.0040(10)$ & $0.0271(11)$ & $0.0141(10)$ \\
\hline $\mathrm{C} 19$ & $0.0424(15)$ & $0.0389(15)$ & $0.0523(18)$ & $0.0006(12)$ & $0.0119(13)$ & $0.0075(13)$ \\
\hline $\mathrm{C} 18$ & $0.0422(15)$ & $0.0405(17)$ & $0.0565(18)$ & $-0.0036(13)$ & $0.0057(13)$ & $0.0064(14)$ \\
\hline $\mathrm{C} 11$ & $0.0478(16)$ & $0.0380(15)$ & $0.0510(17)$ & $-0.0028(13)$ & $0.0001(13)$ & $0.0021(13)$ \\
\hline $\mathrm{C} 16$ & $0.0569(18)$ & $0.0495(17)$ & $0.0538(18)$ & $-0.0046(14)$ & $0.0028(14)$ & $0.0064(14)$ \\
\hline $\mathrm{C} 2$ & $0.0387(15)$ & $0.0391(15)$ & $0.0582(19)$ & $-0.0023(12)$ & 0.0039 & $0.0111(14)$ \\
\hline $\mathrm{C} 3$ & $0.0539(18)$ & $0.0506(18)$ & $0.065(2)$ & $0.0044(15)$ & $0.0038(15)$ & $0.0091(15)$ \\
\hline $\mathrm{C} 12$ & $0.0500(18)$ & 0.0579 (19) & 0.0633 (19) & $-0.0053(15)$ & $0.0046(15)$ & $0.0080(15)$ \\
\hline $\mathrm{C} 7$ & $0.0455(16)$ & $0.0484(17)$ & $0.0611(19)$ & $-0.0051(13)$ & $0.0033(14)$ & $0.0117(15)$ \\
\hline $\mathrm{C} 17$ & $0.068(2)$ & $0.080(2)$ & $0.067(2)$ & $-0.0040(18)$ & $0.0158(17)$ & $0.0151(18)$ \\
\hline $\mathrm{C} 20$ & $0.0559(18)$ & $0.0562(18)$ & $0.0562(18)$ & $-0.0131(15)$ & $0.0101(14)$ & $0.0026(15)$ \\
\hline C6 & $0.0532(18)$ & $0.062(2)$ & $0.058(2)$ & $-0.0064(16)$ & $-0.0075(15)$ & $0.0158(16)$ \\
\hline $\mathrm{C} 24$ & $0.0503(17)$ & $0.0479(17)$ & $0.062(2)$ & $0.0002(14)$ & $0.0069(15)$ & $0.0078(15)$ \\
\hline C34 & $0.065(2)$ & $0.085(2)$ & $0.068(2)$ & 0.0039 (19) & $0.0013(17)$ & 0.0160 (19) \\
\hline $\mathrm{C} 23$ & $0.0582(18)$ & $0.0571(18)$ & $0.0504(18)$ & $0.0051(15)$ & $0.0124(15)$ & $0.0093(15)$ \\
\hline O6 & 0.0905 (18) & $0.0892(18)$ & 0.0804 (17) & $0.0270(16)$ & $-0.0028(14)$ & $-0.0017(14)$ \\
\hline C33 & $0.0586(18)$ & 0.0497 (17) & $0.0571(19)$ & $-0.0006(14)$ & $0.0090(15)$ & $0.0047(15)$ \\
\hline $\mathrm{C} 13$ & 0.0478 (19) & $0.084(2)$ & $0.085(3)$ & $-0.0015(17)$ & $0.0010(17)$ & $0.009(2)$ \\
\hline $\mathrm{C} 22$ & $0.069(2)$ & $0.061(2)$ & $0.068(2)$ & $-0.0093(17)$ & $0.0290(17)$ & $0.0134(17)$ \\
\hline $\mathrm{C} 26$ & $0.060(2)$ & $0.075(2)$ & $0.059(2)$ & $-0.0054(18)$ & $0.0066(17)$ & 0.0032 (19) \\
\hline $\mathrm{C} 4$ & $0.060(2)$ & $0.067(2)$ & $0.090(3)$ & $0.0220(17)$ & 0.0032 (19) & $0.009(2)$ \\
\hline $\mathrm{C} 32$ & $0.085(3)$ & $0.071(2)$ & $0.063(2)$ & -0.0019 (19) & $0.0214(18)$ & $0.0162(18)$ \\
\hline $\mathrm{C} 29$ & 0.0547 (19) & $0.0576(19)$ & $0.073(2)$ & $0.0033(15)$ & $0.0166(16)$ & $0.0071(16)$ \\
\hline $\mathrm{C} 5$ & $0.056(2)$ & $0.071(2)$ & $0.091(3)$ & $0.0117(18)$ & $-0.0102(18)$ & $0.024(2)$ \\
\hline $\mathrm{C} 15$ & $0.069(2)$ & $0.071(2)$ & $0.062(2)$ & $0.0009(18)$ & $-0.0044(17)$ & 0.0197 (17) \\
\hline $\mathrm{C} 21$ & $0.070(2)$ & $0.066(2)$ & $0.070(2)$ & $-0.0283(17)$ & $0.0181(17)$ & $0.0029(18)$ \\
\hline C9 & 0.059 (2) & $0.084(3)$ & $0.074(2)$ & $-0.0010(19)$ & $-0.0024(18)$ & $0.022(2)$ \\
\hline $\mathrm{C} 31$ & $0.071(2)$ & $0.085(3)$ & $0.079(2)$ & $-0.004(2)$ & $0.034(2)$ & $0.014(2)$ \\
\hline $\mathrm{C} 14$ & $0.061(2)$ & $0.079(2)$ & $0.079(2)$ & $0.0102(18)$ & $-0.0053(18)$ & $0.013(2)$ \\
\hline $\mathrm{C} 25$ & $0.074(2)$ & 0.091 (3) & $0.073(2)$ & $-0.036(2)$ & $-0.0032(18)$ & $0.013(2)$ \\
\hline $\mathrm{O} 3$ & 0.097 (2) & $0.108(2)$ & $0.101(2)$ & $-0.0409(18)$ & $0.0092(16)$ & $0.0158(17)$ \\
\hline $\mathrm{C} 30$ & $0.054(2)$ & $0.083(2)$ & $0.093(3)$ & $0.0012(18)$ & 0.0241 (19) & $0.009(2)$ \\
\hline $\mathrm{C} 8$ & $0.080(2)$ & $0.068(2)$ & $0.063(2)$ & $0.0065(18)$ & $0.0068(17)$ & $0.0001(17)$ \\
\hline $\mathrm{C} 10$ & $0.082(3)$ & 0.135 (4) & $0.064(2)$ & -0.010 & $-0.0021(19)$ & $0.020(2)$ \\
\hline $\mathrm{C} 27$ & $0.075(2)$ & $0.119(3)$ & $0.063(2)$ & $-0.005(2)$ & $0.0047(18)$ & $-0.008(2)$ \\
\hline
\end{tabular}

Geometric parameters $\left(\AA,{ }^{\circ}\right)$

\begin{tabular}{llll}
\hline $\mathrm{O} 1-\mathrm{C} 1$ & $1.222(3)$ & $\mathrm{C} 24-\mathrm{C} 25$ & $1.504(4)$ \\
$\mathrm{O} 4-\mathrm{C} 18$ & $1.224(3)$ & $\mathrm{C} 34-\mathrm{C} 33$ & $1.497(4)$ \\
$\mathrm{N} 1-\mathrm{C} 1$ & $1.348(3)$ & $\mathrm{C} 34-\mathrm{H} 34 \mathrm{~A}$ & 0.9600 \\
$\mathrm{O} 3-\mathrm{C} 9$ & $1.186(4)$ & $\mathrm{C} 34-\mathrm{H} 34 \mathrm{~B}$ & 0.9600 \\
$\mathrm{O} 6-\mathrm{C} 26$ & $1.188(4)$ & $\mathrm{C} 34-\mathrm{H} 34 \mathrm{C}$ & 0.9600 \\
$\mathrm{~N} 2-\mathrm{C} 18$ & $1.344(4)$ & $\mathrm{C} 23-\mathrm{C} 22$ & $1.372(4)$ \\
$\mathrm{N} 2-\mathrm{C} 28$ & $1.434(3)$ & $\mathrm{C} 33-\mathrm{C} 32$ & $1.391(4)$
\end{tabular}




\begin{tabular}{|c|c|c|c|}
\hline $\mathrm{N} 2-\mathrm{H} 2$ & $0.88(3)$ & $\mathrm{C} 13-\mathrm{C} 14$ & $1.357(5)$ \\
\hline $\mathrm{O} 2-\mathrm{C} 9$ & $1.365(4)$ & $\mathrm{C} 13-\mathrm{H} 13$ & 0.9300 \\
\hline $\mathrm{O} 2-\mathrm{C} 6$ & $1.414(4)$ & $\mathrm{C} 22-\mathrm{C} 21$ & $1.371(4)$ \\
\hline $\mathrm{C} 1-\mathrm{C} 2$ & $1.499(4)$ & $\mathrm{C} 22-\mathrm{H} 22$ & 0.9300 \\
\hline $\mathrm{N} 1-\mathrm{C} 11$ & $1.427(3)$ & $\mathrm{C} 26-\mathrm{C} 27$ & $1.483(5)$ \\
\hline $\mathrm{N} 1-\mathrm{H} 1$ & $0.91(3)$ & $\mathrm{C} 4-\mathrm{C} 5$ & $1.371(5)$ \\
\hline $\mathrm{O} 5-\mathrm{C} 26$ & $1.359(4)$ & $\mathrm{C} 4-\mathrm{H} 4$ & 0.9300 \\
\hline $\mathrm{O} 5-\mathrm{C} 23$ & $1.409(4)$ & $\mathrm{C} 32-\mathrm{C} 31$ & $1.365(5)$ \\
\hline $\mathrm{C} 28-\mathrm{C} 33$ & $1.378(4)$ & $\mathrm{C} 32-\mathrm{H} 32$ & 0.9300 \\
\hline $\mathrm{C} 28-\mathrm{C} 29$ & $1.400(4)$ & $\mathrm{C} 29-\mathrm{C} 30$ & $1.372(4)$ \\
\hline $\mathrm{C} 19-\mathrm{C} 20$ & $1.378(4)$ & $\mathrm{C} 29-\mathrm{H} 29$ & 0.9300 \\
\hline $\mathrm{C} 19-\mathrm{C} 24$ & $1.399(4)$ & $\mathrm{C} 5-\mathrm{H} 5$ & 0.9300 \\
\hline $\mathrm{C} 19-\mathrm{C} 18$ & $1.502(4)$ & $\mathrm{C} 15-\mathrm{C} 14$ & $1.383(5)$ \\
\hline $\mathrm{C} 11-\mathrm{C} 16$ & $1.384(4)$ & C15-H15 & 0.9300 \\
\hline $\mathrm{C} 11-\mathrm{C} 12$ & $1.400(4)$ & $\mathrm{C} 21-\mathrm{H} 21$ & 0.9300 \\
\hline $\mathrm{C} 16-\mathrm{C} 15$ & $1.387(4)$ & $\mathrm{C} 9-\mathrm{C} 10$ & $1.482(5)$ \\
\hline $\mathrm{C} 16-\mathrm{C} 17$ & $1.501(4)$ & $\mathrm{C} 31-\mathrm{C} 30$ & $1.365(5)$ \\
\hline $\mathrm{C} 2-\mathrm{C} 3$ & $1.384(4)$ & $\mathrm{C} 31-\mathrm{H} 31$ & 0.9300 \\
\hline $\mathrm{C} 2-\mathrm{C} 7$ & $1.404(4)$ & $\mathrm{C} 14-\mathrm{H} 14$ & 0.9300 \\
\hline $\mathrm{C} 3-\mathrm{C} 4$ & $1.385(4)$ & $\mathrm{C} 25-\mathrm{H} 25 \mathrm{~A}$ & 0.9600 \\
\hline $\mathrm{C} 3-\mathrm{H} 3$ & 0.9300 & $\mathrm{C} 25-\mathrm{H} 25 \mathrm{~B}$ & 0.9600 \\
\hline $\mathrm{C} 12-\mathrm{C} 13$ & $1.369(4)$ & $\mathrm{C} 25-\mathrm{H} 25 \mathrm{C}$ & 0.9600 \\
\hline $\mathrm{C} 12-\mathrm{H} 12$ & 0.9300 & $\mathrm{C} 30-\mathrm{H} 30$ & 0.9300 \\
\hline $\mathrm{C} 7-\mathrm{C} 6$ & $1.387(4)$ & $\mathrm{C} 8-\mathrm{H} 8 \mathrm{~A}$ & 0.9600 \\
\hline $\mathrm{C} 7-\mathrm{C} 8$ & $1.497(4)$ & $\mathrm{C} 8-\mathrm{H} 8 \mathrm{~B}$ & 0.9600 \\
\hline C17-H17A & 0.9600 & $\mathrm{C} 8-\mathrm{H} 8 \mathrm{C}$ & 0.9600 \\
\hline C17-H17B & 0.9600 & $\mathrm{C} 10-\mathrm{H} 10 \mathrm{~A}$ & 0.9600 \\
\hline $\mathrm{C} 17-\mathrm{H} 17 \mathrm{C}$ & 0.9600 & $\mathrm{C} 10-\mathrm{H} 10 \mathrm{~B}$ & 0.9600 \\
\hline $\mathrm{C} 20-\mathrm{C} 21$ & $1.383(4)$ & $\mathrm{C} 10-\mathrm{H} 10 \mathrm{C}$ & 0.9600 \\
\hline $\mathrm{C} 20-\mathrm{H} 20$ & 0.9300 & $\mathrm{C} 27-\mathrm{H} 27 \mathrm{~A}$ & 0.9600 \\
\hline $\mathrm{C} 6-\mathrm{C} 5$ & $1.374(5)$ & $\mathrm{C} 27-\mathrm{H} 27 \mathrm{~B}$ & 0.9600 \\
\hline $\mathrm{C} 24-\mathrm{C} 23$ & $1.382(4)$ & $\mathrm{C} 27-\mathrm{H} 27 \mathrm{C}$ & 0.9600 \\
\hline $\mathrm{O} 1-\mathrm{C} 1-\mathrm{N} 1$ & $123.5(3)$ & $\mathrm{C} 32-\mathrm{C} 33-\mathrm{C} 34$ & $120.9(3)$ \\
\hline $\mathrm{O} 4-\mathrm{C} 18-\mathrm{N} 2$ & $123.6(3)$ & $\mathrm{C} 14-\mathrm{C} 13-\mathrm{C} 12$ & $120.1(3)$ \\
\hline $\mathrm{C} 1-\mathrm{N} 1-\mathrm{C} 11$ & $123.4(2)$ & $\mathrm{C} 14-\mathrm{C} 13-\mathrm{H} 13$ & 120.0 \\
\hline $\mathrm{C} 18-\mathrm{N} 2-\mathrm{C} 28$ & $124.2(2)$ & $\mathrm{C} 12-\mathrm{C} 13-\mathrm{H} 13$ & 120.0 \\
\hline $\mathrm{C} 18-\mathrm{N} 2-\mathrm{H} 2$ & $118.5(17)$ & $\mathrm{C} 21-\mathrm{C} 22-\mathrm{C} 23$ & $119.4(3)$ \\
\hline $\mathrm{C} 28-\mathrm{N} 2-\mathrm{H} 2$ & $113.7(17)$ & $\mathrm{C} 21-\mathrm{C} 22-\mathrm{H} 22$ & 120.3 \\
\hline $\mathrm{C} 9-\mathrm{O} 2-\mathrm{C} 6$ & $117.7(2)$ & $\mathrm{C} 23-\mathrm{C} 22-\mathrm{H} 22$ & 120.3 \\
\hline $\mathrm{O} 1-\mathrm{C} 1-\mathrm{C} 2$ & $121.1(2)$ & $\mathrm{O} 6-\mathrm{C} 26-\mathrm{O} 5$ & $122.6(3)$ \\
\hline $\mathrm{N} 1-\mathrm{C} 1-\mathrm{C} 2$ & $115.4(2)$ & $\mathrm{O} 6-\mathrm{C} 26-\mathrm{C} 27$ & $126.8(4)$ \\
\hline $\mathrm{C} 1-\mathrm{N} 1-\mathrm{H} 1$ & $118.2(17)$ & $\mathrm{O} 5-\mathrm{C} 26-\mathrm{C} 27$ & $110.6(3)$ \\
\hline $\mathrm{C} 11-\mathrm{N} 1-\mathrm{H} 1$ & $114.9(17)$ & $\mathrm{C} 5-\mathrm{C} 4-\mathrm{C} 3$ & $119.6(3)$ \\
\hline $\mathrm{C} 26-\mathrm{O} 5-\mathrm{C} 23$ & $118.4(2)$ & $\mathrm{C} 5-\mathrm{C} 4-\mathrm{H} 4$ & 120.2 \\
\hline $\mathrm{C} 33-\mathrm{C} 28-\mathrm{C} 29$ & $121.0(3)$ & $\mathrm{C} 3-\mathrm{C} 4-\mathrm{H} 4$ & 120.2 \\
\hline $\mathrm{C} 33-\mathrm{C} 28-\mathrm{N} 2$ & $122.5(3)$ & $\mathrm{C} 31-\mathrm{C} 32-\mathrm{C} 33$ & $122.1(3)$ \\
\hline $\mathrm{C} 29-\mathrm{C} 28-\mathrm{N} 2$ & $116.5(2)$ & $\mathrm{C} 31-\mathrm{C} 32-\mathrm{H} 32$ & 118.9 \\
\hline
\end{tabular}




\begin{tabular}{|c|c|c|c|}
\hline $\mathrm{C} 20-\mathrm{C} 19-\mathrm{C} 24$ & $121.4(2)$ & $\mathrm{C} 33-\mathrm{C} 32-\mathrm{H} 32$ & 118.9 \\
\hline $\mathrm{C} 20-\mathrm{C} 19-\mathrm{C} 18$ & $119.9(3)$ & $\mathrm{C} 30-\mathrm{C} 29-\mathrm{C} 28$ & $119.8(3)$ \\
\hline $\mathrm{C} 24-\mathrm{C} 19-\mathrm{C} 18$ & $118.7(2)$ & $\mathrm{C} 30-\mathrm{C} 29-\mathrm{H} 29$ & 120.1 \\
\hline $\mathrm{O} 4-\mathrm{C} 18-\mathrm{C} 19$ & $121.1(3)$ & $\mathrm{C} 28-\mathrm{C} 29-\mathrm{H} 29$ & 120.1 \\
\hline $\mathrm{N} 2-\mathrm{C} 18-\mathrm{C} 19$ & $115.3(2)$ & $\mathrm{C} 4-\mathrm{C} 5-\mathrm{C} 6$ & $119.6(3)$ \\
\hline $\mathrm{C} 16-\mathrm{C} 11-\mathrm{C} 12$ & $120.3(3)$ & $\mathrm{C} 4-\mathrm{C} 5-\mathrm{H} 5$ & 120.2 \\
\hline $\mathrm{C} 16-\mathrm{C} 11-\mathrm{N} 1$ & $122.5(2)$ & $\mathrm{C} 6-\mathrm{C} 5-\mathrm{H} 5$ & 120.2 \\
\hline $\mathrm{C} 12-\mathrm{C} 11-\mathrm{N} 1$ & $117.1(2)$ & $\mathrm{C} 14-\mathrm{C} 15-\mathrm{C} 16$ & $121.3(3)$ \\
\hline $\mathrm{C} 11-\mathrm{C} 16-\mathrm{C} 15$ & $117.9(3)$ & $\mathrm{C} 14-\mathrm{C} 15-\mathrm{H} 15$ & 119.4 \\
\hline $\mathrm{C} 11-\mathrm{C} 16-\mathrm{C} 17$ & $121.8(3)$ & $\mathrm{C} 16-\mathrm{C} 15-\mathrm{H} 15$ & 119.4 \\
\hline $\mathrm{C} 15-\mathrm{C} 16-\mathrm{C} 17$ & $120.3(3)$ & $\mathrm{C} 22-\mathrm{C} 21-\mathrm{C} 20$ & $119.8(3)$ \\
\hline $\mathrm{C} 3-\mathrm{C} 2-\mathrm{C} 7$ & $121.3(3)$ & $\mathrm{C} 22-\mathrm{C} 21-\mathrm{H} 21$ & 120.1 \\
\hline $\mathrm{C} 3-\mathrm{C} 2-\mathrm{C} 1$ & $119.0(3)$ & $\mathrm{C} 20-\mathrm{C} 21-\mathrm{H} 21$ & 120.1 \\
\hline $\mathrm{C} 7-\mathrm{C} 2-\mathrm{C} 1$ & $119.6(2)$ & $\mathrm{O} 3-\mathrm{C} 9-\mathrm{O} 2$ & $121.8(3)$ \\
\hline $\mathrm{C} 4-\mathrm{C} 3-\mathrm{C} 2$ & $120.1(3)$ & $\mathrm{O} 3-\mathrm{C} 9-\mathrm{C} 10$ & $127.5(4)$ \\
\hline $\mathrm{C} 4-\mathrm{C} 3-\mathrm{H} 3$ & 119.9 & $\mathrm{O} 2-\mathrm{C} 9-\mathrm{C} 10$ & $110.7(3)$ \\
\hline $\mathrm{C} 2-\mathrm{C} 3-\mathrm{H} 3$ & 119.9 & $\mathrm{C} 30-\mathrm{C} 31-\mathrm{C} 32$ & $120.2(3)$ \\
\hline $\mathrm{C} 13-\mathrm{C} 12-\mathrm{C} 11$ & $120.1(3)$ & $\mathrm{C} 30-\mathrm{C} 31-\mathrm{H} 31$ & 119.9 \\
\hline $\mathrm{C} 13-\mathrm{C} 12-\mathrm{H} 12$ & 119.9 & $\mathrm{C} 32-\mathrm{C} 31-\mathrm{H} 31$ & 119.9 \\
\hline $\mathrm{C} 11-\mathrm{C} 12-\mathrm{H} 12$ & 119.9 & $\mathrm{C} 13-\mathrm{C} 14-\mathrm{C} 15$ & $120.2(3)$ \\
\hline $\mathrm{C} 6-\mathrm{C} 7-\mathrm{C} 2$ & $116.1(3)$ & $\mathrm{C} 13-\mathrm{C} 14-\mathrm{H} 14$ & 119.9 \\
\hline $\mathrm{C} 6-\mathrm{C} 7-\mathrm{C} 8$ & $121.5(3)$ & $\mathrm{C} 15-\mathrm{C} 14-\mathrm{H} 14$ & 119.9 \\
\hline $\mathrm{C} 2-\mathrm{C} 7-\mathrm{C} 8$ & $122.4(3)$ & $\mathrm{C} 24-\mathrm{C} 25-\mathrm{H} 25 \mathrm{~A}$ & 109.5 \\
\hline $\mathrm{C} 16-\mathrm{C} 17-\mathrm{H} 17 \mathrm{~A}$ & 109.5 & $\mathrm{C} 24-\mathrm{C} 25-\mathrm{H} 25 \mathrm{~B}$ & 109.5 \\
\hline $\mathrm{C} 16-\mathrm{C} 17-\mathrm{H} 17 \mathrm{~B}$ & 109.5 & $\mathrm{H} 25 \mathrm{~A}-\mathrm{C} 25-\mathrm{H} 25 \mathrm{~B}$ & 109.5 \\
\hline $\mathrm{H} 17 \mathrm{~A}-\mathrm{C} 17-\mathrm{H} 17 \mathrm{~B}$ & 109.5 & $\mathrm{C} 24-\mathrm{C} 25-\mathrm{H} 25 \mathrm{C}$ & 109.5 \\
\hline $\mathrm{C} 16-\mathrm{C} 17-\mathrm{H} 17 \mathrm{C}$ & 109.5 & $\mathrm{H} 25 \mathrm{~A}-\mathrm{C} 25-\mathrm{H} 25 \mathrm{C}$ & 109.5 \\
\hline $\mathrm{H} 17 \mathrm{~A}-\mathrm{C} 17-\mathrm{H} 17 \mathrm{C}$ & 109.5 & $\mathrm{H} 25 \mathrm{~B}-\mathrm{C} 25-\mathrm{H} 25 \mathrm{C}$ & 109.5 \\
\hline $\mathrm{H} 17 \mathrm{~B}-\mathrm{C} 17-\mathrm{H} 17 \mathrm{C}$ & 109.5 & $\mathrm{C} 31-\mathrm{C} 30-\mathrm{C} 29$ & $119.7(3)$ \\
\hline $\mathrm{C} 19-\mathrm{C} 20-\mathrm{C} 21$ & $119.9(3)$ & $\mathrm{C} 31-\mathrm{C} 30-\mathrm{H} 30$ & 120.2 \\
\hline $\mathrm{C} 19-\mathrm{C} 20-\mathrm{H} 20$ & 120.0 & $\mathrm{C} 29-\mathrm{C} 30-\mathrm{H} 30$ & 120.2 \\
\hline $\mathrm{C} 21-\mathrm{C} 20-\mathrm{H} 20$ & 120.0 & $\mathrm{C} 7-\mathrm{C} 8-\mathrm{H} 8 \mathrm{~A}$ & 109.5 \\
\hline $\mathrm{C} 5-\mathrm{C} 6-\mathrm{C} 7$ & $123.1(3)$ & $\mathrm{C} 7-\mathrm{C} 8-\mathrm{H} 8 \mathrm{~B}$ & 109.5 \\
\hline $\mathrm{C} 5-\mathrm{C} 6-\mathrm{O} 2$ & $118.2(3)$ & $\mathrm{H} 8 \mathrm{~A}-\mathrm{C} 8-\mathrm{H} 8 \mathrm{~B}$ & 109.5 \\
\hline $\mathrm{C} 7-\mathrm{C} 6-\mathrm{O} 2$ & $118.6(3)$ & $\mathrm{C} 7-\mathrm{C} 8-\mathrm{H} 8 \mathrm{C}$ & 109.5 \\
\hline $\mathrm{C} 23-\mathrm{C} 24-\mathrm{C} 19$ & $116.4(3)$ & $\mathrm{H} 8 \mathrm{~A}-\mathrm{C} 8-\mathrm{H} 8 \mathrm{C}$ & 109.5 \\
\hline $\mathrm{C} 23-\mathrm{C} 24-\mathrm{C} 25$ & $121.7(3)$ & $\mathrm{H} 8 \mathrm{~B}-\mathrm{C} 8-\mathrm{H} 8 \mathrm{C}$ & 109.5 \\
\hline $\mathrm{C} 19-\mathrm{C} 24-\mathrm{C} 25$ & $121.8(3)$ & $\mathrm{C} 9-\mathrm{C} 10-\mathrm{H} 10 \mathrm{~A}$ & 109.5 \\
\hline $\mathrm{C} 33-\mathrm{C} 34-\mathrm{H} 34 \mathrm{~A}$ & 109.5 & $\mathrm{C} 9-\mathrm{C} 10-\mathrm{H} 10 \mathrm{~B}$ & 109.5 \\
\hline C33-C $34-\mathrm{H} 34 \mathrm{~B}$ & 109.5 & $\mathrm{H} 10 \mathrm{~A}-\mathrm{C} 10-\mathrm{H} 10 \mathrm{~B}$ & 109.5 \\
\hline $\mathrm{H} 34 \mathrm{~A}-\mathrm{C} 34-\mathrm{H} 34 \mathrm{~B}$ & 109.5 & $\mathrm{C} 9-\mathrm{C} 10-\mathrm{H} 10 \mathrm{C}$ & 109.5 \\
\hline $\mathrm{C} 33-\mathrm{C} 34-\mathrm{H} 34 \mathrm{C}$ & 109.5 & $\mathrm{H} 10 \mathrm{~A}-\mathrm{C} 10-\mathrm{H} 10 \mathrm{C}$ & 109.5 \\
\hline $\mathrm{H} 34 \mathrm{~A}-\mathrm{C} 34-\mathrm{H} 34 \mathrm{C}$ & 109.5 & $\mathrm{H} 10 \mathrm{~B}-\mathrm{C} 10-\mathrm{H} 10 \mathrm{C}$ & 109.5 \\
\hline $\mathrm{H} 34 \mathrm{~B}-\mathrm{C} 34-\mathrm{H} 34 \mathrm{C}$ & 109.5 & $\mathrm{C} 26-\mathrm{C} 27-\mathrm{H} 27 \mathrm{~A}$ & 109.5 \\
\hline $\mathrm{C} 22-\mathrm{C} 23-\mathrm{C} 24$ & $122.9(3)$ & $\mathrm{C} 26-\mathrm{C} 27-\mathrm{H} 27 \mathrm{~B}$ & 109.5 \\
\hline $\mathrm{C} 22-\mathrm{C} 23-\mathrm{O} 5$ & $118.5(3)$ & $\mathrm{H} 27 \mathrm{~A}-\mathrm{C} 27-\mathrm{H} 27 \mathrm{~B}$ & 109.5 \\
\hline $\mathrm{C} 24-\mathrm{C} 23-\mathrm{O} 5$ & $118.4(3)$ & $\mathrm{C} 26-\mathrm{C} 27-\mathrm{H} 27 \mathrm{C}$ & 109.5 \\
\hline $\mathrm{C} 28-\mathrm{C} 33-\mathrm{C} 32$ & $117.0(3)$ & $\mathrm{H} 27 \mathrm{~A}-\mathrm{C} 27-\mathrm{H} 27 \mathrm{C}$ & 109.5 \\
\hline
\end{tabular}




\begin{tabular}{|c|c|}
\hline $\mathrm{C} 28-\mathrm{C} 33-\mathrm{C} 34$ & $122.1(3)$ \\
\hline $\mathrm{C} 9-\mathrm{O} 2-\mathrm{C} 6-\mathrm{C} 7$ & $-100.0(3)$ \\
\hline $\mathrm{N} 1-\mathrm{C} 1-\mathrm{C} 2-\mathrm{C} 7$ & $129.1(3)$ \\
\hline $\mathrm{C} 2-\mathrm{C} 1-\mathrm{N} 1-\mathrm{C} 11$ & $-172.4(2)$ \\
\hline $\mathrm{C} 1-\mathrm{N} 1-\mathrm{C} 11-\mathrm{C} 16$ & $-66.4(4)$ \\
\hline $\mathrm{C} 26-\mathrm{O} 5-\mathrm{C} 23-\mathrm{C} 24$ & $-83.7(3)$ \\
\hline $\mathrm{C} 24-\mathrm{C} 19-\mathrm{C} 18-\mathrm{N} 2$ & $-113.6(3)$ \\
\hline $\mathrm{C} 28-\mathrm{N} 2-\mathrm{C} 18-\mathrm{C} 19$ & $166.2(2)$ \\
\hline $\mathrm{C} 18-\mathrm{N} 2-\mathrm{C} 28-\mathrm{C} 33$ & $66.0(4)$ \\
\hline $\mathrm{N} 1-\mathrm{C} 1-\mathrm{C} 2-\mathrm{C} 3$ & $-53.9(3)$ \\
\hline $\mathrm{O} 1-\mathrm{C} 1-\mathrm{N} 1-\mathrm{C} 11$ & $8.5(4)$ \\
\hline $\mathrm{C} 18-\mathrm{N} 2-\mathrm{C} 28-\mathrm{C} 29$ & $-114.4(3)$ \\
\hline $\mathrm{C} 28-\mathrm{N} 2-\mathrm{C} 18-\mathrm{O} 4$ & $-13.4(4)$ \\
\hline $\mathrm{C} 20-\mathrm{C} 19-\mathrm{C} 18-\mathrm{O} 4$ & $-114.4(3)$ \\
\hline $\mathrm{C} 24-\mathrm{C} 19-\mathrm{C} 18-\mathrm{O} 4$ & $66.0(4)$ \\
\hline $\mathrm{C} 20-\mathrm{C} 19-\mathrm{C} 18-\mathrm{N} 2$ & $66.0(3)$ \\
\hline $\mathrm{C} 1-\mathrm{N} 1-\mathrm{C} 11-\mathrm{C} 12$ & $116.4(3)$ \\
\hline $\mathrm{C} 12-\mathrm{C} 11-\mathrm{C} 16-\mathrm{C} 15$ & $-3.6(4)$ \\
\hline $\mathrm{N} 1-\mathrm{C} 11-\mathrm{C} 16-\mathrm{C} 15$ & $179.2(3)$ \\
\hline $\mathrm{C} 12-\mathrm{C} 11-\mathrm{C} 16-\mathrm{C} 17$ & $173.8(3)$ \\
\hline $\mathrm{N} 1-\mathrm{C} 11-\mathrm{C} 16-\mathrm{C} 17$ & $-3.3(4)$ \\
\hline $\mathrm{O} 1-\mathrm{C} 1-\mathrm{C} 2-\mathrm{C} 3$ & $125.3(3)$ \\
\hline $\mathrm{O} 1-\mathrm{C} 1-\mathrm{C} 2-\mathrm{C} 7$ & $-51.7(4)$ \\
\hline $\mathrm{C} 7-\mathrm{C} 2-\mathrm{C} 3-\mathrm{C} 4$ & $1.3(4)$ \\
\hline $\mathrm{C} 1-\mathrm{C} 2-\mathrm{C} 3-\mathrm{C} 4$ & $-175.6(3)$ \\
\hline $\mathrm{C} 16-\mathrm{C} 11-\mathrm{C} 12-\mathrm{C} 13$ & $3.0(4)$ \\
\hline $\mathrm{N} 1-\mathrm{C} 11-\mathrm{C} 12-\mathrm{C} 13$ & $-179.7(3)$ \\
\hline $\mathrm{C} 3-\mathrm{C} 2-\mathrm{C} 7-\mathrm{C} 6$ & $-0.8(4)$ \\
\hline $\mathrm{C} 1-\mathrm{C} 2-\mathrm{C} 7-\mathrm{C} 6$ & $176.1(2)$ \\
\hline $\mathrm{C} 3-\mathrm{C} 2-\mathrm{C} 7-\mathrm{C} 8$ & $179.1(3)$ \\
\hline $\mathrm{C} 1-\mathrm{C} 2-\mathrm{C} 7-\mathrm{C} 8$ & $-4.0(4)$ \\
\hline $\mathrm{C} 24-\mathrm{C} 19-\mathrm{C} 20-\mathrm{C} 21$ & $-1.4(4)$ \\
\hline $\mathrm{C} 18-\mathrm{C} 19-\mathrm{C} 20-\mathrm{C} 21$ & $179.0(3)$ \\
\hline $\mathrm{C} 2-\mathrm{C} 7-\mathrm{C} 6-\mathrm{C} 5$ & $-0.1(4)$ \\
\hline $\mathrm{C} 8-\mathrm{C} 7-\mathrm{C} 6-\mathrm{C} 5$ & $179.9(3)$ \\
\hline $\mathrm{C} 2-\mathrm{C} 7-\mathrm{C} 6-\mathrm{O} 2$ & $-175.9(2)$ \\
\hline $\mathrm{C} 8-\mathrm{C} 7-\mathrm{C} 6-\mathrm{O} 2$ & $4.1(4)$ \\
\hline $\mathrm{C} 9-\mathrm{O} 2-\mathrm{C} 6-\mathrm{C} 5$ & $84.0(4)$ \\
\hline
\end{tabular}

$\mathrm{H} 27 \mathrm{~B}-\mathrm{C} 27-\mathrm{H} 27 \mathrm{C} \quad 109.5$

$\mathrm{C} 20-\mathrm{C} 19-\mathrm{C} 24-\mathrm{C} 23-0.3$ (4)

$\mathrm{C} 18-\mathrm{C} 19-\mathrm{C} 24-\mathrm{C} 23 \quad 179.3$ (3)

$\mathrm{C} 20-\mathrm{C} 19-\mathrm{C} 24-\mathrm{C} 25 \quad-177.9(3)$

$\mathrm{C} 18-\mathrm{C} 19-\mathrm{C} 24-\mathrm{C} 25 \quad 1.7$ (4)

$\mathrm{C} 19-\mathrm{C} 24-\mathrm{C} 23-\mathrm{C} 22$

$\mathrm{C} 25-\mathrm{C} 24-\mathrm{C} 23-\mathrm{C} 22 \quad 179.4(3)$

$\mathrm{C} 19-\mathrm{C} 24-\mathrm{C} 23-\mathrm{O} 5 \quad-172.5$ (2)

$\mathrm{C} 25-\mathrm{C} 24-\mathrm{C} 23-\mathrm{O} 5 \quad 5.1$ (4)

$\mathrm{C} 26-\mathrm{O} 5-\mathrm{C} 23-\mathrm{C} 22 \quad 101.7$ (3)

$\mathrm{C} 29-\mathrm{C} 28-\mathrm{C} 33-\mathrm{C} 32 \quad 3.9(4)$

$\mathrm{N} 2-\mathrm{C} 28-\mathrm{C} 33-\mathrm{C} 32 \quad-176.5(3)$

$\mathrm{C} 29-\mathrm{C} 28-\mathrm{C} 33-\mathrm{C} 34 \quad-173.8(3)$

$\mathrm{N} 2-\mathrm{C} 28-\mathrm{C} 33-\mathrm{C} 34 \quad 5.8(4)$

$\mathrm{C} 11-\mathrm{C} 12-\mathrm{C} 13-\mathrm{C} 14 \quad-0.3(5)$

$\mathrm{C} 24-\mathrm{C} 23-\mathrm{C} 22-\mathrm{C} 21 \quad-1.6(5)$

$\mathrm{O} 5-\mathrm{C} 23-\mathrm{C} 22-\mathrm{C} 21 \quad 172.7$ (3)

$\mathrm{C} 23-\mathrm{O} 5-\mathrm{C} 26-\mathrm{O} 6 \quad-5.3(5)$

$\mathrm{C} 23-\mathrm{O} 5-\mathrm{C} 26-\mathrm{C} 27 \quad 174.0(3)$

$\mathrm{C} 2-\mathrm{C} 3-\mathrm{C} 4-\mathrm{C} 5 \quad-0.8$ (5)

$\mathrm{C} 28-\mathrm{C} 33-\mathrm{C} 32-\mathrm{C} 31 \quad-2.0(5)$

$\mathrm{C} 34-\mathrm{C} 33-\mathrm{C} 32-\mathrm{C} 31 \quad 175.7$ (3)

$\mathrm{C} 33-\mathrm{C} 28-\mathrm{C} 29-\mathrm{C} 30 \quad-3.0$ (5)

$\mathrm{N} 2-\mathrm{C} 28-\mathrm{C} 29-\mathrm{C} 30 \quad 177.4(3)$

$\mathrm{C} 3-\mathrm{C} 4-\mathrm{C} 5-\mathrm{C} 6 \quad-0.1(5)$

$\mathrm{C} 7-\mathrm{C} 6-\mathrm{C} 5-\mathrm{C} 4 \quad 0.6(5)$

$\mathrm{O} 2-\mathrm{C} 6-\mathrm{C} 5-\mathrm{C} 4 \quad 176.4$ (3)

$\mathrm{C} 11-\mathrm{C} 16-\mathrm{C} 15-\mathrm{C} 14$

$\mathrm{C} 17-\mathrm{C} 16-\mathrm{C} 15-\mathrm{C} 14 \quad-175.9(3)$

$\mathrm{C} 23-\mathrm{C} 22-\mathrm{C} 21-\mathrm{C} 20 \quad-0.1(5)$

$\mathrm{C} 19-\mathrm{C} 20-\mathrm{C} 21-\mathrm{C} 22 \quad 1.6(5)$

$\mathrm{C} 6-\mathrm{O} 2-\mathrm{C} 9-\mathrm{O} 3 \quad 4.8(5)$

$\mathrm{C} 6-\mathrm{O} 2-\mathrm{C} 9-\mathrm{C} 10 \quad-175.4$ (3)

$\mathrm{C} 33-\mathrm{C} 32-\mathrm{C} 31-\mathrm{C} 30 \quad-0.9(6)$

$\mathrm{C} 12-\mathrm{C} 13-\mathrm{C} 14-\mathrm{C} 15 \quad-1.7(5)$

$\mathrm{C} 16-\mathrm{C} 15-\mathrm{C} 14-\mathrm{C} 13 \quad 1.0(5)$

$\mathrm{C} 32-\mathrm{C} 31-\mathrm{C} 30-\mathrm{C} 29 \quad 2.0(6)$

$\mathrm{C} 28-\mathrm{C} 29-\mathrm{C} 30-\mathrm{C} 31 \quad-0.1(5)$

Hydrogen-bond geometry $\left(A,{ }^{\circ}\right)$

$C g 1$ is the centroid of the $\mathrm{C} 28-\mathrm{C} 33$ ring.

\begin{tabular}{lllll}
\hline$D-\mathrm{H} \cdots A$ & $D-\mathrm{H}$ & $\mathrm{H} \cdots A$ & $D \cdots A$ & $D-\mathrm{H} \cdots A$ \\
\hline $\mathrm{C} 5-\mathrm{H} 5 \cdots \mathrm{O} 66^{\mathrm{i}}$ & 0.93 & 2.49 & $3.402(4)$ & 167 \\
$\mathrm{~N} 2-\mathrm{H} 2 \cdots \mathrm{O} 1^{\mathrm{ii}}$ & $0.88(3)$ & $1.96(3)$ & $2.813(3)$ & $164(2)$ \\
$\mathrm{N} 1-\mathrm{H} 1 \cdots \mathrm{O} 4$ & $0.91(3)$ & $1.91(3)$ & $2.804(3)$ & $166(2)$ \\
$\mathrm{C} 25-\mathrm{H} 25 B \cdots \mathrm{O} 4$ & 0.96 & 2.76 & $3.117(4)$ & 103
\end{tabular}


$\mathrm{C} 34-\mathrm{H} 34 A \cdots \mathrm{O} 4$

$\mathrm{C} 8-\mathrm{H} 8 B \cdots \mathrm{O} 1$

$\mathrm{C} 3-\mathrm{H} 3 \cdots \mathrm{Cg} 1$
0.96

0.96

0.93
2.59

2.75

2.81
$3.100(4)$

$2.986(4)$

$3.666(3)$
114

95

153

Symmetry codes: (i) $x-1, y, z$; (ii) $x, y+1, z$. 